
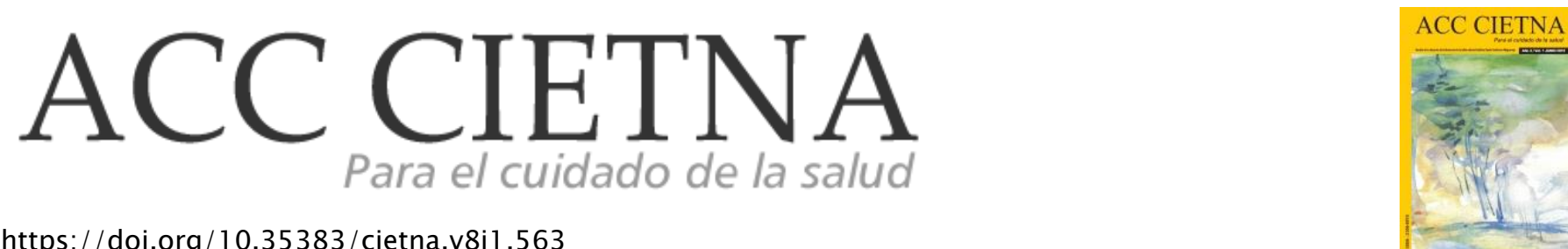

https:// doi.org/10.35383/cietna.v8i1 1.563

\title{
Notificação de agravos e a tomada de decisão de enfermeiros relacionada ao HIV/AIDS
}

\author{
Cunha Machado Tavares Rosangela', Souza Braga André Luiz², Consuelo Ortiz Sanchez Maritza³, \\ Ruiz Barbosa Nassar Pedro4, Lopes de Azevedo Suely 5
}

\begin{tabular}{l} 
INFORMACIÓN DEL ARTÍCULO \\
\hline Historia del artículo: \\
Recibido el 08 de marzo de 2021 \\
Aceptado el 17 de junio de 2021 \\
\hline Palabras clave: \\
Enfermagem \\
HIV \\
Síndrome da Imunodeficiência Adquirida \\
Tomada de Decisão \\
Gerência \\
Sistemas de Informação em Saúde \\
Doenças de Notificação Compulsória
\end{tabular}

Doenças de Notificação Compulsória

\section{RESUMEN}

Objetivo: Refletir sobre a correlação entre os dados dos sistemas de informação em saúde e o processo de tomada de decisão; compreender os conceitos de gerência e gerenciamento aplicados à enfermagem; discutir possíveis implicações para a tomada de decisão na vigilância epidemiológica do HIV/AIDS e as ações de saúde implementadas. Método: Revisão Integrativa de Literatura, realizada entre setembro de 2019 e setembro de 2020 nas bases LILACS, BDENF, MEDLINE/PubMed, Scopus e SciELO. Resultados principais: Identificou-se diferentes percepções quanto aos conceitos de gerência e gerenciamento, notando-se uma influência de concepções pessoais dos autores. O SINAN mostrou que deve ser um dos principais meios para subsidiar o planejamento de ações relacionadas ao HIV/AIDS. Em relação ao agravo, notou-se uma mudança de paradigma acerca das características populacionais na década de 80 e na atual, refletindo uma necessidade de reformulação das políticas de saúde, para que sejam, verdadeiramente, efetivas. Conclusão geral: A tomada de decisão oportuna e com embasamento é primordial na transcendência das barreiras que interferem no planejamento das ações referentes ao agravo apresentado. Necessidade de análise e avaliação rotineira da

\footnotetext{
IEnfermeira Licenciada. Residente em Neonatologia no Instituto Nacional da Mulher, da Criança e do Adolescente Fernandes Figueira da FIOCRUZ, Rio de Janeiro, Brasil. Email: rosangelacmtavares@hotmail.com. ORCID: https://orcid.org/0000-0001-6922-5601

2Doutor em Enfermagem. Professor Adjunto do MFE, Universidade Federal Fluminense, Rio de Janeiro, Brasil. Email: andre.braga@globo.com. ORCID: https://orcid.org/0000-0002-7961-9038

${ }^{3}$ Doutora em Enfermagem. Professora Adjunta do MFE, Universidade Federal Fluminense, Rio de Janeiro, Brasil. Email: morsa_peru@yahoo.com ORCID: https://orcid.org/0000-0002-6123-9846 https://orcid.org/0000-0002-8221-7443

${ }^{4}$ Doutor em Enfermagem. Professor Adjunto do MFE, Universidade Federal Fluminense, Rio de Janeiro, Brasil. Email: pedronassar@id.uff.br ORCID: https://orcid.org/0000-0002-9238-0519

${ }^{5}$ Doutora em Enfermagem. Professora Associada do MFE, Universidade Federal Fluminense, Rio de Janeiro, Brasil. Email: sulazrj@gmail.com. ORCID: https://orcid.org/0000-0003-1107-3427
} 
população, a fim de, além de formular políticas e ações de saúde específicas para cada realidade local, seja também possível conscientizar a sociedade da constante transição populacional da epidemia. Fortalecendo assim, a autonomia, corresponsabilidade e protagonismo dos sujeitos e coletivos, na manutenção da sua saúde.

Notification of grievances and decision-making by nurses related to HIV/AIDS

ABSTRACT

Keywords:

Nursing

HIV

Acquired immunodeficiency syndrome

Decision Making

Management

Health Information Systems

Compulsory Reporting Diseases
Objective: Reflect on the correlation between data from health information systems and the decision-making process; understand the concepts of management and management applied to nursing; discuss possible implications for decision-making in HIV/AIDS epidemiological surveillance and implemented health actions. Method: Integrative Literature Review, carried out between September 2019 and September 2020 in LILACS, BDENF, MEDLINE/PubMed, Scopus and SciELO databases. Main results: Different perceptions regarding the concepts of management and management were identified, noting an influence of the authors' personal conceptions. SINAN showed that it should be one of the main means to support the planning of actions related to HIV/AIDS. Regarding the health problem, a paradigm shift was noted regarding population characteristics in the 1980 s and today, reflecting a need to reformulate health policies so that they are truly effective. Overall conclusion: Timely and well-founded decision-making is essential in transcending the barriers that interfere in the planning of actions related to the presented grievance. Need for routine analysis and assessment of the population, in order, in addition to formulating specific health policies and actions for each local reality, to make society aware of the constant population transition of the epidemic. Thus, strengthening the autonomy, co-responsibility and protagonism of subjects and groups in maintaining their health.

\section{Introducción}

A rotina de um enfermeiro é permeada por diversas atribuições, dentre as quais encontra-se a tomada de decisão, que pode ser compreendida como um encargo que denota o exercício de sua atividade gerencial. E, independente do âmbito que se relacione a sua decisão, esta deve ser produto de um meticuloso processo, que envolva a análise e o estudo dos problemas a partir de um levantamento de dados, a definição de diagnósticos e o estabelecimento de propostas e soluções. De modo a tornar viável sua implementação e para que obtenham-se resultados satisfatórios ${ }^{1}$.

Os Sistemas de Informação em Saúde (SIS) brasileiros, contemplam diversos agravos, e que configuram uma ferramenta auxiliar à tomada decisão dos profissionais de saúde. Em vista disso, há uma preocupação no que concerne à qualidade das informações que os alimentam. Uma vez que estes dados subsidiam o processo decisório dos gestores, auxiliando no planejamento dos programas e políticas, assim como na execução de ações de acordo com cada contexto. 
O Sistema de Informação de Agravos de Notificação (SINAN), recebe os dados relacionados aos agravos considerados de notificação compulsória, incluindo o HIV/AIDS, objeto deste estudo. Segundo dados do Ministério da Saúde², na última década foram notificados, no SINAN, 284.569 casos de infecção pelo vírus HIV, no Brasil, sendo aproximadamente 27.471 no estado do Rio de Janeiro. Havendo entre estes, o predomínio na faixa etária dos 25 aos 29 anos (18,9\%), no gênero masculino $(68,9 \%)$, orientação homossexual $(43,2 \%)$ e com ensino médio incompleto $(15,3 \%)$. Sendo a principal via de transmissão a sexual $(82,0 \%)$ e em menores de 13 anos, a vertical (88\%).

Observa-se, que há uma alta prevalência de HIV/AIDS, atualmente, sugerindo a urgência e a importância de programas e intervenções novas e eficazes na prevenção da infecção ${ }^{3}$. Contudo, a complexidade das informações necessárias para esta reformulação e o tempo limitado dos profissionais na busca de resultados oriundos de pesquisas, tem ressaltado a importância das revisões neste cenário.

Percebe-se que, é necessária melhor organização com estudos de maior rigor metodológico, para garantir a tomada de decisões pelo enfermeiro, com evidências científicas de alta qualidade para melhoria da assistência prestada. Destaca-se também a importância de atualização constante do enfermeiro assistencial na busca, desenvolvimento e utilização de pesquisas na prática profissional, a fim de favorecer a interface entre a pesquisa e o cuidar.

A partir do exposto, nota-se a relevância da informação como subsídio para as ações de saúde. É fundamental que o profissional de enfermagem esteja preparado para avaliar os novos comportamentos do HIV, utilizando-se de suas funções de gestor e educador para promover a prevenção de novos casos e para qualificar a sua prática assistencial neste grupo.
Diante disso, delimitou-se como objetivo geral deste estudo, refletir sobre a correlação entre os dados oriundos dos sistemas de informação em saúde e o processo de tomada de decisão em uma unidade. E como objetivos específicos, compreender os conceitos de gerência e gerenciamento aplicados ao âmbito da enfermagem e discutir possíveis implicações para a tomada de decisão sobre a vigilância epidemiológica do HIV/AIDS e as ações de saúde implementadas em sua região de abrangência.

\section{Metodología}

A fim de obter fundamentação teórica para a elaboração do presente estudo, optou-se pela revisão integrativa da literatura, realizada entre os meses de setembro 2019 e setembro de 2020 .

A partir de então, estabeleceu-se como finalidade a captação de material bibliográfico que fornecesse subsídio para o alcance dos objetivos propostos, pela pesquisa, e que respondessem a seguinte questão: como as informações, notificadas ao SINAN, podem influenciar o processo de tomada de decisão de enfermeiros nas ações relacionadas ao HIV/AIDS?

Uma vez delimitada a questão norteadora, buscaram-se estudos a partir dos seguintes descritores: Enfermagem; HIV; Síndrome de Imunodeficiência Adquirida; Tomada de Decisão; Gerência; Sistemas de Informação em Saúde; Doenças de Notificação Compulsória. Sendo esta fase operacionalizada na Biblioteca Virtual de Saúde (BVS), nas bases de dados Literatura LatinoAmericana em Ciências da Saúde (LILACS) e Base de Dados em Enfermagem (BDENF). Na PubMed, na base Medical Literature Analysis and Retrieval System Online (MEDLINE). No Portal de Periódicos da CAPES, na base Scopus da Elsevier. E, na biblioteca eletrônica Scientific Eletronic Library Online (SCiELO).

O quadro 1 mostra o quantitativo de obras encontradas por descritor nas bases de dados. Posteriormente, associou-se, para refino dos 
resultados, os descritores da seguinte forma: "HIV" AND "Síndrome de Imunodeficiência Adquirida"; "Tomada de Decisão" AND "Enfermagem"; "Gerência" AND "Enfermagem"; "Sistemas de
Informação em Saúde" AND "Doenças de Notificação Compulsória". As referidas associações estão demonstradas no quadro 2 .

Quadro 1 - Quantitativo de obras encontradas por descritor por base de dados

\begin{tabular}{|c|c|c|c|c|c|}
\hline Descritores & LILACS & BDENF & MEDLINE & SciELO & Scopus \\
\hline Enfermagem & 39351 & 30079 & 516275 & 15916 & 15297 \\
\hline HIV & 14328 & 987 & 354273 & 227 & 320598 \\
\hline $\begin{array}{c}\text { Síndrome da Imunodeficiência } \\
\text { Adquirida }\end{array}$ & 10970 & 802 & 80287 & 240 & 168 \\
\hline Tomada de Decisão & 2321 & 327 & 92003 & 1269 & 1198 \\
\hline Gerência & 2283 & 525 & 1870 & 1641 & 2762 \\
\hline Sistemas de Informação em Saúde & 518 & 238 & 1178 & 95 & 127 \\
\hline $\begin{array}{c}\text { Doenças de Notificação } \\
\text { Compulsória }\end{array}$ & 820 & 26 & 4328 & 11 & 16 \\
\hline TOTAL & 70591 & 32984 & 1050214 & 19399 & 340166 \\
\hline
\end{tabular}

Fonte: acervo pessoal, 2020.

Quadro 2 - Associação de descritores e palavras e quantitativo de produções encontradas por base de dados

\begin{tabular}{|c|c|c|c|c|c|}
\hline Descritores & LILACS & BDENF & MEDLINE & SciELO & Scopus \\
\hline $\begin{array}{c}\text { "HIV" AND "Síndrome da } \\
\text { Imunodeficiência Adquirida" }\end{array}$ & 879 & 879 & 181622 & 247 & 163 \\
\hline "Tomada de Decisão" AND "Enfermagem" & 58 & 58 & 06 & 168 & 163 \\
\hline "Gerência" AND "Enfermagem" & 485 & 408 & 184 & 250 & 209 \\
\hline $\begin{array}{c}\text { "Sistemas de Informação em Saúde" AND } \\
\text { "Doenças de Notificação Compulsória" }\end{array}$ & 04 & 04 & 08 & 02 & 02 \\
\hline TOTAL & 1426 & 1349 & 181820 & 667 & 537 \\
\hline
\end{tabular}

Fonte: acervo pessoal, 2020. 
A eleição do material seguiu, inicialmente, alguns critérios de inclusão pré-estabelecidos, foram eles: ter sido publicado entre o hiato temporal do ano de 2015 ao ano de 2020, apresentar-se nos idiomas português, inglês e espanhol e contemplar assuntos que favorecessem uma reflexão acerca da questão norteadora. E, como critério de exclusão, aqueles cuja abordagem não contribuísse com a formulação de respostas para a questão norteadora. Entretanto, em razão da escassez de materiais relacionados a alguns dos assuntos abordados, ampliou-se o hiato temporal para 2010 a 2020

Os estudos encontrados foram selecionados conforme o título sugerisse a abordagem desejada e de acordo com o exposto no resumo. Uma vez que estes atendessem aos critérios de inclusão, eram eleitos, posteriormente lidos e analisados para saber se enquadrar-se-iam ao objetivo da pesquisa. A fim de consolidar o corpus da pesquisa, a busca e análise estendeu-se para livros de acervo pessoal, relacionados à temática estudada.

Após a leitura e análise das produções disponíveis, estas foram selecionadas conforme as temáticas estivessem relacionadas à enfermagem, HIV/AIDS, tomada de decisão e/ou sistemas de informação em saúde, sendo escolhidas - dentre artigos, teses, editoriais e livros - 17 obras, para o desenvolvimento da revisão de literatura.

A prática baseada em evidências preconiza sistemas de classificação, dependendo da abordagem metodológica adotada, que toma por base o delineamento do estudo. Nesta pesquisa, o nivel de evidência dos estudos foi avaliado segundo a classificação do Oxford Centre for Evidence-Based Medicine (Figura 1). Em relação à construção do Fluxograma (Figura 2), para melhor compreensão do caminhar metodológico, optouse por seguir as diretrizes PRISMA.

Figura 1 - Níveis de evidência por tipo de estudo

\begin{tabular}{|c|c|c|c|c|c|}
\hline \multicolumn{6}{|c|}{$\begin{array}{l}\text { Nivel de Evidéncia Cientifica por Tipo de Estudo - "Oxford Centre for } \\
\text { Evidence-based Medicine" }\end{array}$} \\
\hline $\begin{array}{l}\text { Grav de } \\
\text { recomendaçao }\end{array}$ & $\begin{array}{l}\text { Nivel de } \\
\text { evidencis }\end{array}$ & $\begin{array}{l}\text { Tratamento - } \\
\text { Prevençato - Etiologia }\end{array}$ & Prognóstico & Diagnóstico & $\begin{array}{l}\text { Diagnostico Diterencial/ } \\
\text { Prevaléncia de Sintomas }\end{array}$ \\
\hline \multirow{3}{*}{ A } & 1A & $\begin{array}{l}\text { Revisdo sistemasca de } \\
\text { ensavos clinicos } \\
\text { controlados } \\
\text { randomizados }\end{array}$ & $\begin{array}{l}\text { Revisiso Sistemblica de } \\
\text { Coortos desde o inicio } \\
\text { da doensa. Criterio } \\
\text { Prognostico validado } \\
\text { em diversas } \\
\text { populapoes. }\end{array}$ & $\begin{array}{l}\text { Revisto Sistemitica de } \\
\text { estudos diagnósticos nivel } \\
\text { 1. Criberio Diagnóstico de } \\
\text { estudos nivel } 18 \text {. em } \\
\text { difecentes centros clínicos. }\end{array}$ & $\begin{array}{l}\text { Revisdo sistemasca de } \\
\text { estudos de coorte } \\
\text { (contemporanea ou } \\
\text { prospectiva) }\end{array}$ \\
\hline & 18 & $\begin{array}{l}\text { Ensaio dilinico } \\
\text { controlado randomizado } \\
\text { com intervalo de } \\
\text { conflança estreito }\end{array}$ & $\begin{array}{c}\text { Coonte desde o inicio } \\
\text { da doenca, com perda } \\
<20 \% \text {. Criserio } \\
\text { prognosition validado } \\
\text { em uma única } \\
\text { pepulaça. }\end{array}$ & $\begin{array}{l}\text { Coorte validada, com bom } \\
\text { padráo do referéncia. } \\
\text { Critério Diagndstico } \\
\text { testado em um unico } \\
\text { centro clinico. }\end{array}$ & $\begin{array}{l}\text { Estudo de coorte com } \\
\text { poucas perdas }\end{array}$ \\
\hline & 1c & $\begin{array}{l}\text { Resultados terapbuticos } \\
\text { so tpo "tudo ou nada" }\end{array}$ & $\begin{array}{l}\text { Serie de casos do tipo } \\
\text { Tudo ou nada" }\end{array}$ & $\begin{array}{l}\text { Sensibalidade } \\
\text { especiticidade proximas } \\
\text { de took }\end{array}$ & $\begin{array}{l}\text { Serie de casos do tipo } \\
\text { tudo ou nada" }\end{array}$ \\
\hline \multirow{5}{*}{ B } & $2 \mathrm{~A}$ & $\begin{array}{l}\text { Revisho Sistematica de } \\
\text { Estudos de Coorte }\end{array}$ & $\begin{array}{l}\text { Revisao Sistematica de } \\
\text { coortes histcricas } \\
\text { (retrospectivas) ov de } \\
\text { seguimento de casos } \\
\text { nabo fratados de grupo } \\
\text { controle de onsaio } \\
\text { clinico randomizado }\end{array}$ & $\begin{array}{c}\text { Revisso Sistemstica de } \\
\text { estudios diagnósticos de } \\
\text { nivel }>2\end{array}$ & $\begin{array}{l}\text { Revisao Sistematica de } \\
\text { estudos sobre diagnostice } \\
\text { diferencial de nivel }>2\end{array}$ \\
\hline & 28 & $\begin{array}{l}\text { Estudo de Coonte } \\
\text { (Uncluindo Ensaio } \\
\text { clinico Randomizado } \\
\text { de monor qualidade) }\end{array}$ & 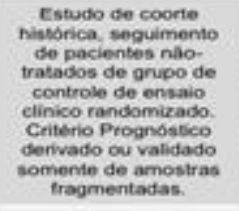 & $\begin{array}{l}\text { Coorte exploratoria com } \\
\text { bom padrao de referencia. } \\
\text { Criterio Oiagndotico } \\
\text { derivado ou validado em } \\
\text { amostras fragmentadas ou } \\
\text { banco de dados }\end{array}$ & $\begin{array}{l}\text { Estudo de coorte historica } \\
\text { ou com seguimento de } \\
\text { casos comprometido } \\
\text { (numero grande de } \\
\text { perdas) }\end{array}$ \\
\hline & $2 \mathrm{c}$ & 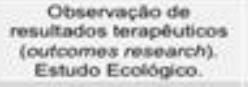 & $\begin{array}{l}\text { Observacalo de } \\
\text { Evolucbes Cinicas } \\
\text { (outcomes research) }\end{array}$ & - & Estudo Ecologico \\
\hline & 3A & $\begin{array}{l}\text { Revisalo Sistematica de } \\
\text { Esturisos Caso-Comrote }\end{array}$ & $-m$ & $\begin{array}{c}\text { Revisaso Sistemstica de } \\
\text { eatudios diagnósticos de } \\
\text { nivel >3B }\end{array}$ & $\begin{array}{l}\text { Revisbo Sistematica de } \\
\text { estudos de nivel >3B }\end{array}$ \\
\hline & 38 & Estudo Caso-Controte & + & 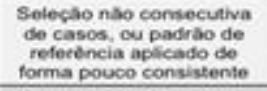 & 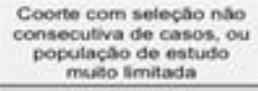 \\
\hline c & 4 & $\begin{array}{l}\text { Relato de Casos } \\
\text { (inchuindo coorte ou } \\
\text { cesso-controle de menor } \\
\text { qualidade) }\end{array}$ & $\begin{array}{l}\text { Serie de casos (e } \\
\text { coorte prognosica de } \\
\text { menor qualidade) }\end{array}$ & $\begin{array}{l}\text { Estudo de caso-controle } \\
\text { ou padrao de referencia } \\
\text { pobre ou nato } \\
\text { independente }\end{array}$ & $\begin{array}{l}\text { Série de casos, ou padrâo } \\
\text { de referencia superado }\end{array}$ \\
\hline D & 5 & & & do com animais) & Basicas \\
\hline
\end{tabular}

Fonte: Oxford Centre for Evidence-Based Medicine: levels of evidence, 2009. 


\section{Resultados, análisis y discusión}

Identificou-se um total de 1.513 .354 artigos, sendo 70.591 na LILCAS, 32.984 na BDENF, 1.050.214 na MEDLINE, 19.399 na SCIELO e 340.166 na SCOPUS. Houve duplicidade em 471 artigos. Foram selecionados para leitura 59 artigos. Destes, 42 foram excluídos por não se referirem à temática em estudo ou não responderem à questão norteadora. A amostra compreendeu 17 estudos, selecionados conforme demonstra o fluxograma (Figura 2).

Figura 2 - Fluxograma explicativo da seleção de artigos. Niterói (RJ), 2020

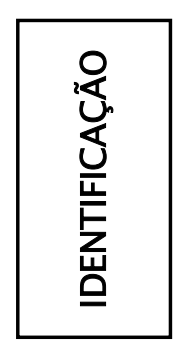

\section{Registros identificados por meio de pesquisas nas bases de dados}

LILACS $(n=70591)$; BDENF $(n=32984)$; MEDLINE $(n=1050214)$; SCIELO $(n=19399)$; SCOPUS $(n=340166)$.
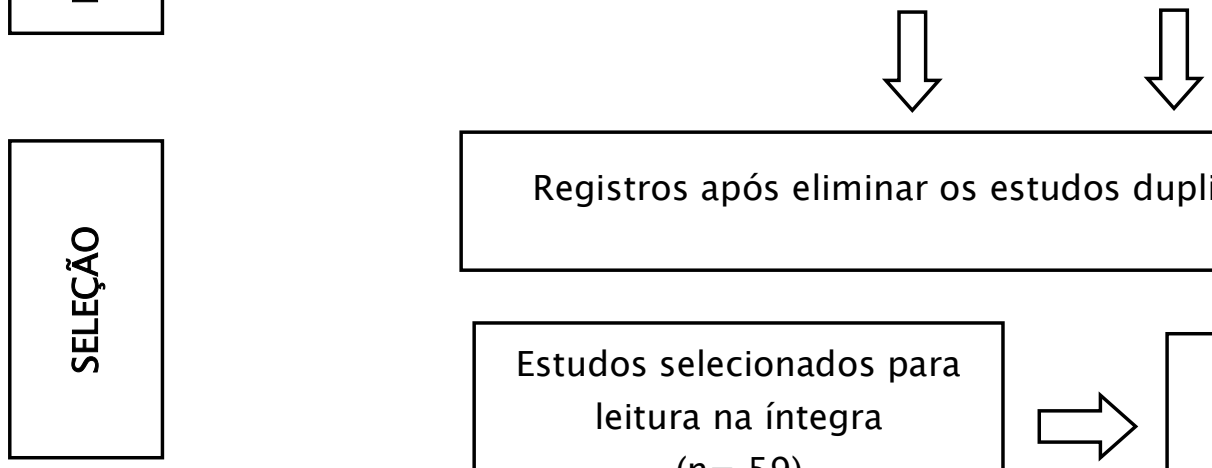

Registros após eliminar os estudos duplicados $(n=145747)$

$$
\begin{aligned}
& \text { Estudos selecionados para } \\
& \text { leitura na íntegra } \\
& (n=59)
\end{aligned}
$$
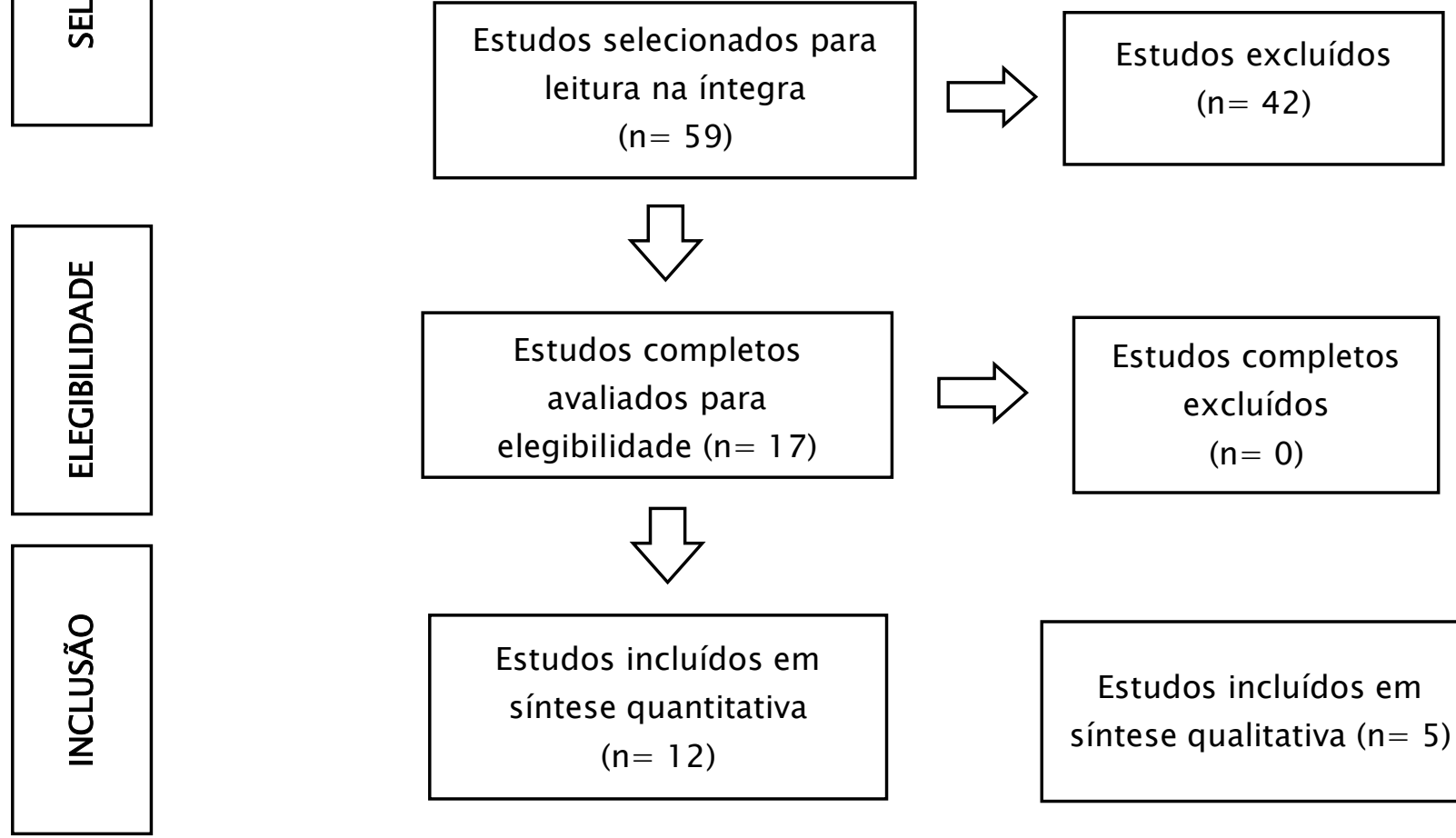

Estudos incluídos em síntese qualitativa $(n=5)$

Fonte: acervo pessoal, 2020.

A partir disto, de acordo com o encontrado na literatura, para dar coerência ao estudo foi verificada a necessidade de versar sobre três categorias que permeiam a tomada de decisão do enfermeiro, a partir da qualidade das informações sobre HIV/AIDS notificadas ao Sistema de Informação de Agravos de Notificação (SINAN). São eles: "Aspectos Gerais do HIV/AIDS"; "Tomada de Decisão e o Gerenciamento da Assistência de Enfermagem"; "O SINAN como Facilitador no Processo de Tomada de Decisão em Relação ao HIV/AIDS". 
O primeiro item selecionado tratou dos aspectos gerais relacionados ao HIV/AIDS, tais como: os biológicos, fisiológicos, históricos e sociais. Além de enfatizar a necessidade de um maior aprofundamento na temática, para um melhor direcionamento de ações preventivas a este agravo.

O segundo, por sua vez, trouxe o conceito de tomada decisão e a inter-relação com as práticas gerenciais nas estruturas organizacionais, sobretudo, no âmbito da saúde e na gestão em enfermagem. Sugerindo, ainda, o assunto do último item, ao versar sobre como o processo decisório pode ser qualificado através da tecnologia da informação,
Por fim, o terceiro contexto traz concepções acerca dos sistemas de informação na área da saúde, com ênfase ao SINAN e às notificações compulsórias de HIV/AIDS, e a correlação da qualidade dos dados gerados nestes sistemas para a tomada decisão em saúde.

\section{Aspectos Gerais do HIV/AIDS}

As discussões apresentadas na presente categoria foram construídas a partir da seleção de cinco publicações, sendo uma de 2010, uma de 2016, uma de 2017, uma de 2018 e uma de 2019. Apresenta-se a seguir, no quadro 3, a relação de autores, ano de publicação e título de cada obra.

Quadro 3 - Relação de artigos selecionados com seus respectivos autores e ano de publicação para a construção da categoría

\begin{tabular}{|c|c|c|c|c|}
\hline Autor (es) & $\begin{array}{l}\text { Periódico/Editora - } \\
\text { Ano }\end{array}$ & Título & Base & $\begin{array}{l}\text { Nível de } \\
\text { Evidência }\end{array}$ \\
\hline $\begin{array}{l}\text { Guerra CPP, Seidl } \\
\text { EMF. }\end{array}$ & $\begin{array}{l}\text { Psicologia em } \\
\text { Estudo - } 2010\end{array}$ & $\begin{array}{l}\text { Adesão em HIV/AIDS: estudo com } \\
\text { adolescentes e seus cuidadores } \\
\text { primários }\end{array}$ & SciELO & $3 B$ \\
\hline $\begin{array}{l}\text { Miziara LAF, } \\
\text { Andrade FMO }\end{array}$ & $\begin{array}{l}\text { Boletim - Academia } \\
\text { Paulista de } \\
\text { Psicologia - } 2016\end{array}$ & $\begin{array}{l}\text { O significado do HIV/Aids na vida } \\
\text { de crianças e adolescentes que } \\
\text { vivem com a doença }\end{array}$ & SciELO & $3 B$ \\
\hline $\begin{array}{l}\text { Vilela WV, } \\
\text { Barbosa RM. }\end{array}$ & $\begin{array}{l}\text { Ciência e Saúde } \\
\text { Coletiva - } 2017\end{array}$ & $\begin{array}{c}\text { Trajetória de mulheres vivendo } \\
\text { com HIV/aids no Brasil. Avanços e } \\
\text { permanência da resposta à } \\
\text { epidemia }\end{array}$ & SciELO & $3 B$ \\
\hline $\begin{array}{l}\text { Silva SRA, } \\
\text { Marques APO, } \\
\text { Leal MCC, et al }\end{array}$ & $\begin{array}{l}\text { Estudos } \\
\text { Interdisciplinares } \\
\text { sobre o } \\
\text { Envelhecimento - } \\
2018\end{array}$ & $\begin{array}{l}\text { Pessoas com } 50 \text { anos e mais com } \\
\text { HIV/AIDS no Brasil: Quem são? }\end{array}$ & LILACS & 4 \\
\hline $\begin{array}{l}\text { Trindade FF, } \\
\text { Fernandes GT, } \\
\text { Nascimento RHF, } \\
\text { et al }\end{array}$ & $\begin{array}{l}\text { Journal Health } \\
\text { NPEPS. } 2019\end{array}$ & $\begin{array}{l}\text { Perfil epidemiológico e análise de } \\
\text { tendência do HIV/AIDS }\end{array}$ & LILACS & $3 \mathrm{~A}$ \\
\hline
\end{tabular}

Fonte: acervo pessoal, 2020.

A Síndrome da Imunodeficiência Humana (AIDS) é uma doença infectocontagiosa causada pelo Vírus da Imunodeficiência Humana (HIV), que leva à perda progressiva da imunidade, sendo resultado 
da diminuição da taxa dos linfócitos CD4 no indivíduo infectado, culminando consequentemente, com 0 aumento de sua vulnerabilidade à outras doenças.

Descoberta mundialmente no início da década de 1980, a AIDS tornou-se um marco histórico da humanidade, comportando-se de forma epidêmica. No Brasil, os primeiros casos ocorreram em 1982, sobretudo nas regiões metropolitanas de São Paulo e Rio de Janeiro.

Os casos de HIV/AIDS desde o seu surgimento caracterizavam-se pelas seguintes características: pessoas do sexo masculino, alto nível socioeconômico, pertencentes às categorias de transmissão homossexuais/bissexuais, além de portadores de hemofilia ou em receptores de sangue ${ }^{4}$ Entretanto, frente a multidimensionalidade da infecção vive-se no cenário atual, principalmente, os processos de feminização, pauperização e interiorização dos casos de AIDS.

Para Vilela e Barbosa5 grande parte das mulheres não se reconhece como pertencente a grupos e comportamentos considerados "de risco", o que acaba por dificultar a adoção de tecnologias de proteção e de medidas de enfrentamento adaptativo.

No que se refere à interiorização e pauperização, se na década de 1980 , os casos estavam centrados nos círculos das grandes metrópoles. Atualmente, caracteriza-se por, também, afetar indivíduos que residem em pequenos municípios, mais pobres, com poucos recursos financeiros e com uma população com baixo nível de escolaridade. Expressando uma problemática referente ao acesso e a qualidade das políticas públicas de prevenção, controle da disseminação e assistência.

Ao longo da revisão bibliográfica, foi possível observar que há uma gama de estudos sobre a população idosa vivendo com HIV/AIDS, entretanto, no que se refere à criança e ao adolescente, estes são escassos. Notou-se, na faixa etária idosa, assim como supracitado em relação às mulheres, que parte desta não se reconhecia como "grupo de risco" antes de adquirir a doença, outros não pensavam no assunto $e$ aqueles em relacionamento fixo afirmaram confiança no (a) parceiro (a). Atualmente, a maior soroprevalência encontra-se entre os 60 e 75 anos, sexo masculino, solteiros e heterossexuais.

No que tende às crianças e adolescentes, a maioria dos estudos enfatizam as questões de aceitabilidade e convivência com o diagnóstico. Destaca-se que para este grupo o sigilo é uma das principais estratégias de enfrentamento, a fim de evitar o preconceito por parte das pessoas com as quais se relacionam.

Os sentimentos de medo e ansiedade, para estes, correlacionam-se principalmente à lembrança de sua condição em razão da tomada diária da medicação, à iminência de uma internação e/ou efeitos adversos do tratamento e, ainda, ao que diz respeito à sexualidade em futuros relacionamentos. E isto, por sua vez, afeta negativamente a autoestima e a percepção acerca de si mesmos. Entretanto, apesar desse conjunto de questões, buscam viver de forma natural, como pessoas que não possuem a doença6.

Ainda sobre crianças e adolescentes, observa-se a influência do papel do cuidado na adesão (ou não) destes ao tratamento. Entretanto, apesar disso, muitas vezes há falta de comunicação no ambiente familiar em relação à condição sorológica da criança. Podendo gerar aumento da sua vulnerabilidade e dificuldade de enfrentamento ${ }^{7}$.

Portanto, a partir do que fora apresentado, é possível concluir que mesmo diante de um cenário mais favorável, quando comparado à outrora, o desenvolvimento de políticas de saúde pública direcionadas e realmente efetivas ainda faz-se necessário na prevenção e controle da epidemia de HIV/AIDS. Uma vez que existem diversas particularidades que tornam cada grupo vivendo com HIV/AIDS único, independente de compartilharem o mesmo diagnóstico. 
É importante perceber que o binômio permanece como importante preocupação, mesmo diante dos avanços nas medidas de prevenção primária e secundária - como incentivo ao uso de preservativos, criação dos Centros de Testagem e Aconselhamento, para diagnóstico precoce, e fornecimento gratuito da terapia antirretroviral. Talvez, um dos grandes desafios atuais, seja a falta de compreensão sobre particularidades epidemiológicas da doença, que analisadas de forma global não conseguem direcionar intervenções locais ${ }^{8}$.
Tomada de Decisão e o Gerenciamento da Assistência em Enfermagem

$\mathrm{Na}$ presente categoria apresentar-se-á o conceito de "tomada de decisão" e suas implicações na gerência em saúde, sobretudo, a realizada pelo enfermeiro. Como subsídio para o desenvolvimento das questões que permeiam a temática em questão foram utilizadas seis obras sendo uma de 2011 , uma de 2012 , uma de 2015 , duas de 2017 e uma de 2019 . No quadro 4 estão relacionados os artigos utilizados.

Quadro 4 - Relação de artigos selecionados com seus respectivos autores e ano de publicação para a construção da categoria

\begin{tabular}{|c|c|c|c|c|}
\hline Autor(es) & $\begin{array}{c}\text { Periódico/Editora } \\
\text { - Ano }\end{array}$ & Título & Base & $\begin{array}{l}\text { Nível de } \\
\text { Evidência }\end{array}$ \\
\hline Ribeiro JMS. & $\begin{array}{c}\text { Revista de } \\
\text { Enfermagem } \\
\text { Referência - } 2011\end{array}$ & $\begin{array}{c}\text { Autonomia Profissional dos } \\
\text { Enfermeiros }\end{array}$ & SciELO & $3 \mathrm{~A}$ \\
\hline $\begin{array}{l}\text { Christovam BP, } \\
\text { Porto IS, Oliveira } \\
\text { DC. }\end{array}$ & $\begin{array}{l}\text { Revista da Escola } \\
\text { de Enfermagem } \\
\text { da USP - } 2012\end{array}$ & $\begin{array}{l}\text { Nursing care management in } \\
\text { hospital settings: the bulding of a } \\
\text { construct. }\end{array}$ & SciELO & 4 \\
\hline $\begin{array}{l}\text { Eduardo EA, Peres } \\
\text { AM, Almeida LM, et } \\
\text { al }\end{array}$ & $\begin{array}{c}\text { Revista Brasileira } \\
\text { de Enfermagem - } \\
2015\end{array}$ & $\begin{array}{l}\text { Análise do modelo de tomada de } \\
\text { decisão de enfermeiros gerentes: } \\
\text { uma reflexão coletiva }\end{array}$ & SciELO & 4 \\
\hline Braga ALS. & $\begin{array}{l}\text { Universidade } \\
\text { Federal } \\
\text { Fluminense - } \\
2017\end{array}$ & $\begin{array}{l}\text { O cotidiano da prática do enfermeiro } \\
\text { de rede básica de saúde: } \\
\text { reflexões/ações sobre a informação } \\
\text { para a tomada de decisão }\end{array}$ & $\begin{array}{l}\text { LILACS, } \\
\text { BDENF }\end{array}$ & $3 \mathrm{~A}$ \\
\hline $\begin{array}{l}\text { Santos PR, Silva SV, } \\
\text { Rigo DFH, et al }\end{array}$ & $\begin{array}{l}\text { Ciência, Cuidado } \\
\text { e Saúde - } 2017\end{array}$ & $\begin{array}{l}\text { Ensino do gerenciamento e suas } \\
\text { implicações à formação do } \\
\text { enfermeiro: perspectivas dos } \\
\text { docentes }\end{array}$ & LILACS & 4 \\
\hline $\begin{array}{l}\text { Tenório HAA, } \\
\text { Souza IB, Júnior } \\
\text { ELG, et al. }\end{array}$ & $\begin{array}{c}\text { Revista de } \\
\text { Enfermagem UFPE } \\
-2019\end{array}$ & $\begin{array}{l}\text { Gestão e Gerenciamento de } \\
\text { Enfermagem: perspectivas de } \\
\text { atuação do discente }\end{array}$ & BDENF & $3 B$ \\
\hline
\end{tabular}

Fonte: acervo pessoal, 2020.

Os moldes do gerenciamento em enfermagem podem contextualizados desde a segunda metade do século XIX, a partir do trabalho de Florence
Nightingale na Guerra da Crimeia, com a organização do cuidado e direção do ambiente onde ficavam grande parte dos feridos e enfermos da guerra. Nota-se desde então que, mesmo 
diante de um cenário desfavorável, mudanças importantes podem ocorrer quando há uma boa administração dos recursos disponíveis.

O conceito de gerenciar no âmbito da enfermagem é, por muitas vezes, pouco compreendido pelo profissional. E fora possível perceber, através da análise da literatura, uma divergência de opiniões entre os próprios autores que conceituam estes termos.

Para Santos et aP, no trabalho em enfermagem, gerenciamento/gerência pode ser compreendido como cuidado indireto, sendo um subprocesso que embasa diversas áreas de prática do enfermeiro, compreendendo a elaboração de estratégias racionais buscando melhores condições de assistência de enfermagem e de trabalho da equipe.

Enquanto para Christovam et al ${ }^{10}$ a retórica acerca do conhecimento relacionado à gerência, constróise em um arranjo de relações entre os diversos entendimentos intrínsecos a este conceito e sua interface com a prática profissional do enfermeiro. De modo que, a aplicabilidade deste conjunto ao cotidiano, envolve uma gama de competências e habilidades para, de fato, acontecer.

Compreende-se, a partir da supracitada contextualização e de uma reflexão crítica, que de forma geral, gestão e gerência podem ser encarados como expressões equânimes, indicando ações e decisões que organizam o trabalho em enfermagem.

No que tende às decisões todos necessitam tomálas, sejam como indivíduos ou como administradores, e isso tem estreita relação com o aproveitamento de oportunidades e com a competência para resolver problemas ${ }^{1}$. No âmbito da saúde, os enfermeiros passam a maior parte de seu tempo analisando situações e tomando decisões. Decidir em um curto espaço de tempo é um ponto-chave nas organizações. Contudo, questões complexas fazem parte do universo que envolve 0 processo decisório, como 0 embasamento em experiências passadas, influência de valores e crenças, habilidades e conhecimento prévio do decisor ${ }^{12}$.

Diante disso, desenvolver aptidões para a tomada de decisão é essencial para a excelência da prática profissional e para a qualidade dos serviços de saúde. Para tal, os enfermeiros dispõem de uma ampla gama de elementos que subsidiam e determinam suas ações. Percebe-se que, no mundo pós-moderno, a aquisição da competência gerencial é possível, e deve basear-se nos ensinamentos produzidos pela experiência individual a partir das vivências do serviço em que se atua ${ }^{13}$.

Para Ribeiro ${ }^{14}$, a autonomia profissional é um atributo essencial ao enfermeiro para assumir a responsabilidade das decisões tomadas. O autor analisou diversas variáveis que poderiam influenciar o profissional de enfermagem, em sua autonomia, e inferiu que as que demonstraram maior correlação positiva foram: capacidade de tomar decisões, habilitações acadêmicas, grau de categoria profissional e satisfação com a profissão.

Diante do supracitado, é possível destacar que embora essencial e imprescindível na prática profissional do enfermeiro, uma vez que este é gestor do cuidado, a tomada de decisão e o gerenciamento são movimentos complexos que requerem aprimoramento constante para ser efetivo, a fim de minimizar os possíveis erros, influenciados pelas características pessoais do tomador de decisões.

\section{O SINAN como Facilitador no Processo de Tomada de Decisão em Relação ao HIV/AIDS}

A fim de elucidar algumas características do SINAN e como estas, junto com outros determinantes, instrumentalizam a tomada de decisão em saúde, o presente contexto utilizou-se de um total seis obras para o seu desenvolvimento sendo uma de 2011 , uma de 2012 , uma de 2013 , uma de 2014 e duas de 2016. O supracitado encontra-se referenciado, abaixo, no quadro 5 . 
Quadro 5 - Relação de artigos selecionados com seus respectivos autores e ano de publicação para a construção da categoria

\begin{tabular}{|c|c|c|c|c|}
\hline Autor(es) & $\begin{array}{c}\text { Periódico/Editora/Equipe } \\
\text { Editorial - Ano }\end{array}$ & Título & Base & $\begin{array}{l}\text { Nível de } \\
\text { Evidência }\end{array}$ \\
\hline $\begin{array}{l}\text { Vidor AC, Fisher } \\
\text { PD, Bordin R. }\end{array}$ & $\begin{array}{l}\text { Revista de Saúde Pública } \\
\qquad-2011\end{array}$ & $\begin{array}{l}\text { Utilização dos sistemas de } \\
\text { informação em saúde em } \\
\text { municípios gaúchos de } \\
\text { pequeno porte }\end{array}$ & SciELO & $3 A$ \\
\hline $\begin{array}{l}\text { Barreto PA, } \\
\text { Braga ALS, } \\
\text { Andrade } \mathrm{M} \text {. }\end{array}$ & $\begin{array}{l}\text { Online Brazilian Journal } \\
\text { of Nursing - } 2012\end{array}$ & $\begin{array}{l}\text { Avaliação da completitude } \\
\text { dos registros de dengue: } \\
\text { estudo exploratório das } \\
\text { notificações compulsórias }\end{array}$ & LILACS & $3 B$ \\
\hline $\begin{array}{l}\text { Angelotti LCZ, } \\
\text { Alexandre PBD, } \\
\text { Miranzi SSC, et } \\
\text { al }\end{array}$ & $\begin{array}{l}\text { Revista de Enfermagem e } \\
\text { Atenção à Saúde - } 2013\end{array}$ & $\begin{array}{l}\text { Qualidade de dados de } \\
\text { informação e } \\
\text { acompanhamento dos casos } \\
\text { de tuberculose em Minas } \\
\text { Gerais }\end{array}$ & BDENF & $3 A$ \\
\hline $\begin{array}{l}\text { Bartholomay P, } \\
\text { Oliveira } \\
\text { GP, Pinheiro RS, } \\
\text { Vasconcelos } \\
\text { ANM. }\end{array}$ & $\begin{array}{l}\text { Cadernos de Saúde } \\
\text { Pública - } 2014\end{array}$ & $\begin{array}{l}\text { Melhoria da qualidade das } \\
\text { informações sobre } \\
\text { tuberculose a partir do } \\
\text { relacionamento entre bases } \\
\text { de dados }\end{array}$ & SCIELO & $3 A$ \\
\hline $\begin{array}{l}\text { Pinheiro ALS, } \\
\text { Andrade KTS, } \\
\text { Silva, DO, et al }\end{array}$ & $\begin{array}{l}\text { Texto \& Contexto } \\
\text { Enfermagem - } 2016\end{array}$ & $\begin{array}{l}\text { Gestão da saúde: o uso dos } \\
\text { sistemas de informação e o } \\
\text { Compartilhamento de } \\
\text { Conhecimento para a Tomada } \\
\text { de Decisão }\end{array}$ & SCIELO & $3 B$ \\
\hline $\begin{array}{l}\text { Júnior SHAS, } \\
\text { Mota JC, SILVA, } \\
\text { RS, et al }\end{array}$ & $\begin{array}{l}\text { Epidemiologia e Serviços } \\
\text { de Saúde - } 2016\end{array}$ & $\begin{array}{l}\text { Descrição dos registros } \\
\text { repetidos no Sistema de } \\
\text { Informação de Agravos de } \\
\text { Notificação, Brasil, 2008-2009 }\end{array}$ & MEDLINE & $3 A$ \\
\hline
\end{tabular}

Fonte: acervo pessoal, 2020.

O Sistema de Informação de Agravos de Notificação (SINAN) objetiva a coleta e processamento dos dados sobre agravos de notificação compulsória em todo o território nacional e configura uma das principais fontes de informação para vigilância epidemiológica no Brasil, detectando surtos e/ou epidemias, bem como elaborando hipóteses epidemiológicas ${ }^{15}$.
A entrada de dados no SINAN, ocorre através da Ficha Individual de Notificação (FIN) e a da Ficha Individual de Investigação (FII). A infecção pelo HIV/AIDS faz parte da Lista Nacional de Notificação Compulsória de doenças, agravos e eventos de saúde pública (Anexo b), que é obrigatória aos médicos e outros profissionais de saúde que atuam na assistência ao paciente. 
Notifica-se como caso confirmado de HIV/AIDS, todo indivíduo que apresentar evidência laboratorial da infecção pelo HIV (dois testes de triagem de detecção de anticorpos anti-HIV ou um confirmatório reagente) e, além disso, um somatório de pelo menos dez pontos numa escala de sinais, sintomas ou doenças, independentemente da presença de outras causas de imunodeficiência. Sendo a notificação realizada, por um profissional capacitado, através do preenchimento da FIN².

No que refere ao SINAN, enquanto SIS, este pode ser qualificado a partir de múltiplas dimensões. Atributos como duplicidade, cobertura, completitude e confiabilidade são relacionados à acurácia das informações. Enquanto, sobre registros repetidos, a falta de correção é um obstáculo à qualidade da notificação, podendo gerar superestimação dos coeficientes de incidência e de prevalência de uma doença ${ }^{16}$.

Com isso, observa-se que a excelência de um SIS depende diretamente da qualidade dos dados que o subsidiam. E que esta que pode ser comprometida quando os formulários não são adequadamente preenchidos ou há lacunas em sua produção e gerenciamento ${ }^{17}$. Portanto, é infactível não notar que a qualidade das informações se mostra uma importante limitação para a análise dos dados dos sistemas de informação em saúde, podendo gerar avaliações equivocadas e, com isto, comprometer o processo decisório.

Entende-se que para o SINAN estabelecer sua hegemonia como fonte de dados, para os agravos considerados de notificação compulsória, é mandatória a conscientização de cada profissional envolvido sobre sua responsabilidade na cadeia produtiva. A fim de que a partir disso, haja ampla cobertura e qualidade nas informações que alimentarão o sistema, garantindo a integralidade nas ações que serão por estas subsidiadas e, consequentemente, a melhoria nas condições de saúde e de vida da população ${ }^{18}$.
Vidor et al ${ }^{19}$ concordam que a preocupação com a formação e qualificação dos recursos humanos é fator preponderante tanto nas questões objetivas como nas subjetivas, e podem significar um fator importante para a subutilização dos SIS.

Conclui-se, portanto, que os SIS se bem utilizados constituem uma ferramenta efetiva na tomada de decisão e no gerenciamento da assistência de enfermagem, sobretudo no que concerne às ações relacionadas ao HIV/AIDS, que muitas vezes - em razão da amplitude de seus determinantes ultrapassam os muros das instituições de saúde.

Refletir-se-á acerca da fala de Pinheiro et aRo, que afirmam que não há uma fórmula que leve a se efetivar a utilização dos SIS para a tomada de decisão pelos gestores da saúde. Todavia, é válido que a gestão promova o fortalecimento de uma cultura informacional e um ambiente organizacional que consolide o uso da informação para a construção do conhecimento e o seu compartilhamento para subsidiar o processo decisório.

\section{Conclusiones}

Através da presente pesquisa foi possível compreender que a tomada de decisão oportuna e com embasamento, pelo profissional de saúde, é primordial na transcendência das barreiras que interferem no planejamento das ações referentes ao agravo apresentado.

Identificou-se, através da análise dos estudos, diferentes percepções quanto aos conceitos de gerência e gerenciamento. Notando-se uma influência de concepções pessoais dos autores, ainda que complementares, na definição destes termos. Esta dualidade, quando na prática, pode interferir no processo decisório de gestores e para isto observou-se a necessidade da aquisição de competências e habilidades que os fortaleça na tomada de decisão, além de subsídios de qualidade para tal. 
Observou-se que o SINAN pode e deve ser um dos principais meios para subsidiar o planejamento de ações relacionadas ao HIV/AIDS. Entretanto, diversos estudos concordam que são necessários atributos relacionados à qualidade dos dados como confiabilidade, cobertura e, principalmente, completitude - além daqueles intrínsecos ao tomador de decisões.

Notou-se o quão influente é o fator humano na alimentação dos SIS e o quanto é sine qua non o comprometimento dos profissionais de saúde na utilização das ferramentas disponíveis - como o preenchimento das fichas de notificação compulsória do SINAN - para que se compreenda a situação real, permitindo clareza na tomada de decisão.

No que tende aos estudos relacionados diretamente à temática "HIV/AIDS", estes maximizam a comparação entre as características das pessoas vivendo com HIV/AIDS na década de 80 e atualmente, e como essa mudança de paradigma reflete uma necessidade de reformulação das políticas de saúde, para que sejam, verdadeiramente, efetivas. Uma vez que, na sociedade contemporânea, coexistem populações vivendo com a infecção com necessidades diferentes.

Conclui-se, que embora descoberto há mais de 40 anos, o HIV/AIDS ainda é permeado por um estigma obsoleto de quem é seu portador e grupo de risco. E este, dificulta a adoção de medidas de prevenção. Diante disso, faz-se necessária a análise e avaliação rotineira da população a fim de, além de formular (e reformular) políticas e ações de saúde específicas para cada realidade local, seja também possível conscientizar a sociedade da constante transição populacional da epidemia. Fomentando assim, a autonomia, corresponsabilidade e protagonismo dos sujeitos e coletivos na manutenção da sua saúde.

\section{Bibliografía}

1. Marquis BL, Huston CJ. Administração e Liderança em Enfermagem. $6^{a}$ ed. Porto Alegre: Artmed, 2015

2. Brasil. Ministério da Saúde, Secretaria de Vigilância em Saúde. Departamento de Vigilância, Prevenção e Controle das Infecções Sexualmente Transmissíveis, do HIV/Aids e das Hepatites Virais. Boletim Epidemiológico Aids/DST [internet]. 2019 [Acesso 10 Mai 2020]; 5(1). Disponível em: http://www.aids.gov.br/pt$\mathrm{br} / \mathrm{pub} / 2019 /$ boletim-epidemiologico-dehivaids-2019

3. Tetteh RA, Yankey BA, Nartey ET, et al. PreExposure Prophylaxis for HIV Prevention: Safety Concerns. Drug Safety [internet]. 2017 [Acesso 10 Mai 2020];40(4):273-283. Disponível em: https://dx.doi.org/10.1007/s40264-0170505-6

4. Silva SRA, Marques APO, Leal MCC, et al. Pessoas com 50 anos e mais com HIV/AIDS no Brasil: Quem são?. Estudos Interdisciplinares sobre Envelhecimento [internet]. 2018 [Acesso 10 Mai 2020]; 23(2): 149-165. Disponível em: https://seer.ufrgs.br/RevEnvelhecer/article/v iew $/ 75018 / 52452$

5. Villela WV, Barbosa RM. Trajetória de mulheres vivendo com HIV/ aids no Brasil. Avanços e permanências da resposta à epidemia. Ciência \& Saúde Coletiva [internet]. 2017 [Acesso 31 Set 2019]; 22(1): 87-96. Disponível em: https://dx.doi.org/10.1590/1413812320172 21.14222016

6. Miziara LAF, Andrade FMO. O significado do HIV/Aids na vida de crianças e adolescentes que vivem com a doença. Boletim-Academia Paulista de Psicologia [internet]. 2016 [Acesso 20 Dec 2019]; 36(90). Disponível em: http://pepsic.bvsalud.org/scielo.php?script= sci_arttext\&pid=S1415-

$711 \times 2016000100003$

7. Guerra CPP, Seidl EMF. Adesão em HIV/AIDS: estudo com adolescentes e seus cuidadores 
primários. Psicologia em Estudo [internet]. 2010 [Acesso 20 Dec 2019]; 15(4). Disponível em: https://www.scielo.br/scielo.php?pid=S1413 =

\section{$\underline{73722010000400014 \& \text { script }=\text { sci_arttext } \& \text { tln }}$ $\mathrm{g}=\mathrm{pt}$}

8. Trindade FF, Fernandes GT, Nascimento RHF, et al. Perfil epidemiológico e análise de tendência do HIV/AIDS. Journal Health NPEPS [internet]. 2019 [Acesso 20 Dec 2019]; 4(1):153-165. Disponível em: https://docs.bvsalud.org/biblioref/2019/06/ 999663/3394-12867-2-pb.pdf

9. Santos PR, Silva SV, Rigo DFH, et al. Ensino do gerenciamento e suas implicações à formação do enfermeiro: perspectivas de docentes. Ciência, Cuidado e Saúde [internet]. 2017 [Acesso 31 Oct 2019]. Disponível em: www.periodicos.uem.br/ojs/! index.php/CiencCuidSaude/article/view/333 $81 / 19004$

10. Christovam BP, Porto IS, Oliveira DC. Nursing care management in hospital settings: the bulding of a construct. Revista Escola de Enfermagem da USP [internet]. 2012 [Acesso 31 Oct 2019]; 46(3):734-41. Disponível em: https://www.scielo.br/j/reeusp/a/5FVLGNZM 4kHDGkFKMdhRtMM/?lang $=$ en\&format $=$ pdf

11. Braga ALS. O cotidiano da prática do enfermeiro de rede básica de saúde: reflexões/ações sobre a informação para a tomada de decisão [Tese Doutorado em Ciências do Cuidado em internet]. Niterói: Universidade Federal Fluminense; 2017 [Acesso 31 Oct 2019]. 197p. Disponível em: https://app.uff.br/riuff/bitstream/1/4007/1 Andre\%20Luiz\%20de\%20Souza\%20Braga.pdf

12. Eduardo EA, Peres AM, Almeida LM, et al. Análise de modelo de tomada de decisão de enfermeiros gerentes: uma reflexão coletiva. Revista Brasileira de Enfermagem [internet]. 2015 [Acesso 31 Oct 2019]; 68(4): 668-75. Disponível em: https://www.scielo.br/j/reben/a/wzd3wPLxy R94vnvdmwP6mWK/?lang $=\mathrm{pt}$
13. Tenório HAA, Souza IB, Júnior ELG, et al. Gestão e Gerenciamento de Enfermagem: perspectivas de atuação do discente. Revista de Enfermagem UFPE [internet]. 2019 [Acesso 20 Dec 2019]; 13: e240535. Disponível em: https://periodicos.ufpe.br/revistas/revistaenf ermagem/article/view/240535/32955

14. Ribeiro JMS. Autonomia Profissional dos Enfermeiros. Revista de Enfermagem Referência [internet]. 2011 [Acesso 20 Dec 2019]; 3(5). Disponível em: https://www.redalyc.org/pdf/3882/3882399 64004.pdf

15. Angelotti LCZ, Alexandre PBD, Miranzi SSC, et al. Qualidade de dados de informação e acompanhamento dos casos de tuberculose em Minas Gerais. Revista Enfermagem e Atenção à Saúde [internet]. 2013 [Acesso 20 Dec 2019]; 2(2): 84-9. Disponível em: http://seer.uftm.edu.br/revistaeletronica/ind ex.php/enfer/article/view/387/410

16. Júnior SHAS, Mota JC, Silva RS, et al. Descrição dos registros repetidos no Sistema de Informação de Agravos de Notificação, Brasil, 2008-2009. Epidemiologia e Serviços de Saúde [internet]. 2016 [Acesso 20 Dec 2019]; 25 (3). Disponível em: https://www.scielo.br/j/ress/a/jXgpYGNjJj8K v6QckxzYKwK/abstract/?lang $=p t$

17. Bartholomay P, Oliveira GP, Pinheiro RS, Vasconcelos ANM. Melhoria da qualidade das informações sobre tuberculose a partir do relacionamento entre bases de dados. Cadernos de Saúde Pública [internet]. 2014 [Acesso 20 Dec 2019]; 30(11). Disponível em: https://www.scielo.br/j/csp/a/vnrBPjQNXgrt zVMVhTZMQtr/?lang=pt

18. Barreto PA, Braga ALS, Andrade M. Avaliação da completitude dos registros de dengue: estudo exploratório das notificações compulsórias. Online Brazilian Journal of Nursing [internet]. 2012 [Acesso 10 Sep 2019]; Disponível em: http://www.objnursing.uff.br/index.php/nur sing/article/view/3920/html_2 
19. Vidor AC, Fisher PD, Bordin R. Utilização dos sistemas de informação em saúde em municípios gaúchos de pequeno porte. Revista de Saúde Pública [internet]. 2011 [Acesso Ago 2020]; 45(1). Disponível em: https://www.scielo.br/scielo.php?script $=$ sci_ arttext\&pid $=$ S0034-89102011000100003

20. Pinheiro ALS, Andrade KTS, Silva DO, et al. Gestão da saúde: o uso dos sistemas de informação e o Compartilhamento de Conhecimento para a Tomada de Decisão. Texto \& Contexto Enfermagem [internet]. 2016 [Acesso Ago 2020]; 25(3): e3440015. Disponível em: https://www.scielo.br/j/tce/a/Wpt3XsBswvC dWPtz8k4MpXJ/?lang=pt 